\title{
Metallomics
}

Cite this: Metallomics, 2014, 6, 921

Received 23rd January 2014, Accepted 17th February 2014

DOI: $10.1039 / c 4 m t 00022 f$

www.rsc.org/metallomics

\section{Manganese leads to an increase in markers of oxidative stress as well as to a shift in the ratio of $\mathrm{Fe}(\mathrm{II}) /(\mathrm{III})$ in rat brain tissue}

\author{
Katharina Fernsebner, *a Julia Zorn, ${ }^{b}$ Basem Kanawati, ${ }^{a}$ Alesia Walker ${ }^{a}$ and \\ Bernhard Michalke ${ }^{a}$
}

\begin{abstract}
Occupationally or environmentally caused chronic exposure to Manganese ( $\mathrm{Mn}$ ) can lead to a degeneration of dopaminergic neurons inducing a Parkinson-like complaint called manganism. Deciphering the ongoing neurodegenerative mechanisms in the affected brain is still a major task for understanding the complex modes of action. Therefore, we applied a non-toxic, oral feeding in rats simulating a chronic exposure to $\mathrm{Mn}$. Analysis of brain extracts by electrospray ionization Fourier transform resonance mass spectrometry (ESI-FT-ICR-MS) revealed an increase in markers of oxidative stress like glutathione disulfide (GSSG), prostaglandins, and 15(S)-HETE, a marker of lipid peroxidation. Furthermore, acetylcholinesterase (AchE) activity and glutamate concentrations were elevated in brain samples of $\mathrm{Mn}$-supplemented rats, suggesting oxidative stress in the brain tissue. Application of ion chromatography coupled to inductively coupled plasma-optical emission spectrometry (IC-ICP-OES) further showed a shift of Fe(III) towards Fe(II) in the brain samples enabling for example the action of the Fenton reaction. This is the first time that changes in the Fe-species distribution could be related to $\mathrm{Mn}$-induced neuroinflammation and is therefore enlarging the knowledge of this complex neurodegenerative condition. The combination of our findings provides substantial evidence that $\mathrm{Mn}$-induced neuroinflammation leads to oxidative stress triggered by multifactorial pathophysiological processes.
\end{abstract}

\section{Introduction}

Manganese (Mn) is an essential trace element, important for numerous physiological processes such as bone and connective tissue growth or amino acid, lipid and carbohydrate metabolism. ${ }^{1}$ This element functions as a cofactor of antioxidative enzymes including Mn superoxide dismutase or acetylcholine esterase (AchE). Thus, it is also involved in protection of cells against oxidative stress. Moreover, Mn plays an important role in the synthesis and transport of neurotransmitters, e.g. serving as a cofactor of glutamine synthetase. ${ }^{2,3}$ At physiological concentrations Mn contributes to the appropriate function of the mentioned processes, while an overexposure is associated with neurotoxic effects resulting in a disease termed manganism.

Humans have a higher risk due to inappropriate protection when occupationally exposed to $\mathrm{Mn} .{ }^{4-6}$ In particular, welders or

\footnotetext{
${ }^{a}$ Research Unit Analytical Biogeochemistry, Helmholtz Zentrum München - German Research Center for Environmental Health ( $\mathrm{GmbH})$, Ingolstädter Landstraße 1, D-85764 Neuherberg, Germany.

E-mail: Katharina.Fernsebner@helmholtz-muenchen.de

${ }^{b}$ Research Unit Comparative Medicine, Helmholtz Zentrum München - German Research Center for Environmental Health (GmbH), Ingolstädter Landstraße 1, D-85764 Neuherberg, Germany
}

miners are accounted to the high-risk group, respirating certain amounts of Mn-dust almost every day, ${ }^{7}$ which can contribute to certain health problems also post-occupationally. ${ }^{8}$ Moreover, as part of a worldwide increase in industrial emission ${ }^{9}$ or the use of organically bound $\mathrm{Mn}$ in pesticides and in MMT (methylcyclopentadienyl Mn tricarbonyl) as an anti-knock agent in gasoline, humans are also getting environmentally exposed to Mn leading to combined exposure scenarios. ${ }^{9}$

Along with overexposure, $\mathrm{Mn}$ has been shown to cross the cerebrospinal fluid barrier and to accumulate in the area of the basal ganglia. ${ }^{10}$ This is known to lead to psychological and motor disturbances similar to symptoms observed in Parkinson Disease (PD). ${ }^{11}$ Mechanisms for Mn-induced neurotoxicity are varied with mitochondrial inactivation as the primary route of action. ${ }^{12}$ This results in enhanced production of reactive oxygen species (ROS) in the affected brain areas leading to oxidative stress. $^{13}$

The PD-like effects of manganism are thought to arise from the damage of output pathways downstream from the nigrostriatal dopaminergic pathway. ${ }^{14}$ In the striatum, dopamine along with $\gamma$-aminobutyric acid (GABA) controls motor behaviors, while their afferents arise from cortical glutamate, the major excitatory neurotransmitter. ${ }^{15,16}$ Therefore, Erikson et al. postulated that 
abnormal striatal glutamate (and/or GABA) metabolism may indirectly contribute to $\mathrm{Mn}$-induced neurotoxicity in dopaminergic pathways. ${ }^{14}$ So far, it seems as if Mn disrupts glutamate transport systems resulting in reduced glutamate uptake and elevated extracellular glutamate concentrations primarily observed in astrocytes. ${ }^{17}$

Furthermore, several studies reported about an altered AchE activity due to Mn-substitution. AchE is an essential enzyme in the central nervous system (CNS), promoting the catalysis and transmission of the neurotransmitter acetylcholine in cholinergic synapses, involved in the regulation of intracellular calcium levels, neurite outgrowth and in neuronal survival. ${ }^{18}$ Therefore, AchE inhibitors are used for treatment of neurodegenerative disorders like Alzheimer's Disease (AD) ${ }^{19}$ In general, determination of AchE activity is often used for prediction of induced neurotoxicity as is the case with high Mn exposure. Nevertheless, it still remains unclear whether Mn-substitution leads to an increase or decrease in AchE activity, as Mn intoxication might act in a time and/or dose-dependent manner.

Based on this background, the aim of this study was to explore the effects of oral exposure of rats to Mn lasting several weeks as a model for chronic Mn exposure. The Mn feeding levels were adjusted for the induction of Mn-induced neuroinflammation. To elucidate the different mechanisms likely responsible for Mn-induced neuroinflammation, we determined both glutamate levels and AchE activity in rat brain extracts. Additionally, targeted metabolite analysis of rat brain samples by electrospray ionization Fourier transform ion cyclotron resonance mass spectrometry (ESI-FT-ICR-MS) was performed to obtain a deeper understanding of neurotoxic mechanisms due to Mn substitution. Such an approach was used to unravel possible markers of neuroinflammation or oxidative stress.

For further investigation of the molecular mechanism of Mn-overexposure, we determined $\mathrm{Fe}(\mathrm{II}) /(\mathrm{III})$ ratios in the rat brain. Several neurodegenerative diseases such as AD, Huntington Disease, amyotrophic lateral sclerosis and also PD were associated with brain Fe accumulation with highest Fe concentrations observed in globus pallidus and substantia nigra amongst other brain areas. ${ }^{20}$ $\mathrm{Fe}$, predominantly in the form of $\mathrm{Fe}(\mathrm{II})$, is known to catalyze the formation of reactive oxygen species (e.g. hydroxyl radical) and the initiation or enhancement of lipid peroxidation by reacting with hydrogen peroxide via the Fenton reaction. ${ }^{21}$ Hence, Fe can act as a highly neurotoxic agent. ${ }^{22}$ So far, there have been only a few studies on the relation between $\mathrm{Fe}$ and manganism. It is noteworthy that $\mathrm{Mn}$ is primarily elevated in Fe-rich brain regions during manganism and that Fe-deficiency can enhance $\mathrm{Mn}$ uptake into the brain. ${ }^{23,24}$ However, determination of different Fe oxidation states during Mn exposure has - to our knowledge - never been an objective of previous studies. Thus, our study will enhance current knowledge of ongoing Mn-related neurodegenerative mechanisms.

\section{Material and methods}

\section{Chemicals}

The multi-elemental standard solution for quantification of elements by ICP-OES or ICP-sf-MS was purchased from SCP
Science (Quebec, Canada) and rhodium (Rh) from Spex CertiPrep (NJ, USA). $\mathrm{FeCl}_{2} \cdot 4 \mathrm{H}_{2} \mathrm{O}$ was purchased from AppliChem $\mathrm{GmbH}$ (Darmstadt, Germany) and Ferric Citrate from Sigma Aldrich Chemie (Steinheim, Germany). Substances for eluent preparation were: Tris, purchased from Carl Roth GmbH + Co. KG (Karlsruhe, Germany), $\mathrm{NH}_{4} \mathrm{Ac}$ from Merck (Darmstadt, Germany) and pyridinedicarbonic acid (PDCA) from Sigma Aldrich Chemie (Steinheim, Germany). $\mathrm{HNO}_{3}$ was purchased from Merck (Darmstadt, Germany) as $65 \%$ nitric acid for analysis and purified by sub-boiling distillation (Berghof, Mühlhausen, Germany). $\mathrm{MeOH}$ for extraction for measurement at FT-ICR-MS was purchased from Lichrosolv, Sigma-Aldrich Chemie (Steinheim, Germany). The purity of used chemicals was $\geq 99.9 \%$ throughout.

\section{Animals and treatment}

12 male Sprague-Dawley rats (RjHan:SD) were purchased from Janvier (Janvier S.A.S., France) directly after weaning at three weeks of age to ensure sustenance by only mother's milk. The animals were kept in pairs in polycarbonate cages type III under specified pathogen-free conditions at a 12/12 hours light cycle. The cages were embedded with hemp mats to facilitate quantitative collection of feces for analysis. Paper houses were given as environmental enrichment. The whole experiment lasted 53 days. All animals were supplemented with filtered tap water and fodder ad libitum over the whole experimental period. For the feeding experiment the control group $(n=6)$ received a standard diet with $23 \mathrm{mg}$ Mn per $\mathrm{kg}$ fodder whilst the test group $(n=6)$ received the same but Mn enriched fodder (500 $\mathrm{mg} \mathrm{kg}{ }^{-1}$ ), which is still in the range of recommended feeding according to NRC nutrient requirements of laboratory animals. ${ }^{25}$ Euthanasia was performed by cutting through the aorta abdominalis suprarenalis after deep narcotization with $5 \%$ isoflurane and total loss of consciousness. All procedures herein were in accordance with the institutional Animal Welfare Committee as well as the German regulations for experimental animals treatment. The brains were taken, weighed and frozen immediately in liquid nitrogen until sample preparation. All applied preparation instruments were made of ceramic to prevent elemental contamination of tissue.

\section{Nitric acid digestion of whole brain and measurement by ICP-OES}

For determination of whole elemental concentrations in the brain tissue, brains were roughly homogenized under liquid nitrogen using a PTFE pistil and $100 \mathrm{mg}$ were transferred into quartz vessels $(n=3) .1 \mathrm{~mL}$ of distilled nitric acid was added to each glass and these were installed into a Seiff apparatus. The digestion was carried out overnight at $176{ }^{\circ} \mathrm{C}$ in a dryer. The digested samples were filled up with aqua bidest to the $10 \mathrm{~mL}$ mark the next day and measured by ICP-OES (Optima 7300 DV, Perkin Elmer, Germany). A certified multi-elemental standard was applied for quantification. Determination of $\mathrm{Mn}$ was performed on $257.611 \mathrm{~nm}$ and of Fe on $259.941 \mathrm{~nm}$, respectively. Sample introduction was performed using a peristaltic pump (0.65 $\left.\mathrm{mL} \mathrm{min}^{-1}\right)$, connected to a Meinhard nebulizer fitted into a cyclone spray chamber. Read parameters were 4 points per peak, 
with 2 replicates and a delay time of 60 seconds. Plasma conditions were set to $15 \mathrm{~L} \mathrm{Ar}$ per min for plasma gas and $0.6 \mathrm{~L} \mathrm{Ar}$ per min for nebulizer gas at a RF power of $1200 \mathrm{~W}$. Blank and standard solutions were measured at the beginning, at the end and in the middle (between control and test samples) of the sample solutions.

Quality control. For quality control, certified reference material (bovine liver, BCR 185; $n=3$ ) was digested and measured in a row with the samples. The recovery obtained for Fe was $203 \pm$ $3.5 \mu \mathrm{g} \mathrm{g}^{-1}$ and for Mn $8.1 \pm 0.01 \mu \mathrm{g} \mathrm{g}^{-1}$ (declared values: Fe: $214 \pm$ $\left.5 \mu \mathrm{g} \mathrm{g}^{-1}, \mathrm{Mn}: 9.3 \pm 0.3 \mu \mathrm{g} \mathrm{g}^{-1}\right)$.

\section{Extraction of brain tissue}

Extraction for $\mathrm{Fe}(\mathrm{II}) /(\mathrm{III})$-speciation, determination of AchE activity and glutamate levels. The extraction of brain tissue was carried out according to the method developed by Diederich et al. for specific extraction of $\mathrm{Mn}$ and $\mathrm{Fe}^{26}{ }^{26}$ For eliminating the risk of oxidation, the extraction was carried out in a glove bag (Atmos Bag, Sigma-Aldrich) under an Ar atmosphere on ice. In short, deep frozen brains were roughly grinded under liquid nitrogen. $0.5-1 \mathrm{~g}$ of the rough homogenate was transferred into $4 \mathrm{~mL}$ of ice cold extraction buffer $(10 \mathrm{mM}$ Tris- $\mathrm{HCl}, \mathrm{pH} 7.4$, previously purged with helium for $3 \mathrm{~h}$ ). After transfer and homogenization in a $10 \mathrm{~mL}$ glass homogenizer (Fortuna, Neolab, Germany), the homogenate was centrifuged in two steps ( 5 minutes at $1434 \times g$ at RT and 45 minutes at $20160 \times g$ at $2{ }^{\circ} \mathrm{C}$, Biofuge 17 RS, Heraeus-Sepatech, Osterode). The supernatant was transferred into an eppendorf tube while the pellet was resuspended in extraction buffer and centrifuged again (30 minutes at $20160 \times g$ at $2{ }^{\circ} \mathrm{C}$ ). The supernatant was added to the one obtained before and the extract was deep-frozen at $-80{ }^{\circ} \mathrm{C}$ in aliquots until analysis. The pellet was kept at $4{ }^{\circ} \mathrm{C}$ until microwave digestion (Microwave3000, Anton Paar, Austria). For the microwave digestion, the pellet was transferred into the quartz glass of the microwave device with $5 \mathrm{~mL}$ of distilled nitric acid. The used program was: $0-5 \mathrm{~W}$ for 5 minutes, $500 \mathrm{~W}$ for 10 minutes, $500-1000 \mathrm{~W}$ for 5 minutes, $1000 \mathrm{~W}$ for 45 minutes and back to $0 \mathrm{~W}$ within 15 minutes. After cooling of the cases the digested samples were filled up to $50 \mathrm{~mL}$ with aqua bidest and stored at room temperature until analysis. For each brain two extractions were performed yielding 12 extracts per group.

Extraction for ESI-FT-ICR-MS measurement. The extracts used in the ESI-FT-ICR-MS measurement were prepared differently to the one used for speciation as the focus in this measurement was on whole molecules and any kind of salt would interfere with the experimental conditions. For each roughly homogenized brain (under liquid nitrogen, see above), $50 \mathrm{mg}$ were diluted with $500 \mu \mathrm{L}$ of $50 \% \mathrm{MeOH}$ and sonicated on ice for 20 minutes. The samples were transferred into a $2 \mathrm{~mL}$ glass homogenizer (Fortuna, Neolab, Germany) with $250 \mu \mathrm{L}$ of $50 \%$ $\mathrm{MeOH}$. After homogenization the extract was transferred back into the eppendorf tube with $250 \mu \mathrm{L}$ of $50 \% \mathrm{MeOH}$ and again sonicated on ice for 20 minutes. After centrifugation for 30 minutes at $2{ }^{\circ} \mathrm{C}$ and $18900 \times g$, the supernatant was diluted with $70 \% \mathrm{MeOH}(1: 10)$. The extracts were stored at $-80{ }^{\circ} \mathrm{C}$ until measurement with ESI-FT-ICR-MS.

\section{ICP-sf-MS and ICP-OES analyses of brain extracts and pellets}

For determination of elemental concentrations of $\mathrm{Mn}$ in brain extracts and pellets ICP-sf-MS analysis was carried out due to low elemental concentrations (Element 2, Thermo Fisher Scientific, Germany). Brain extracts were thawed in the refrigerator overnight and diluted 1:10 with aqua bidest and the acid digested pellets were measured undiluted. In both cases, $1 \mu \mathrm{g} \mathrm{L}^{-1} \mathrm{Rh}$ was used as the internal standard. Plasma conditions were $15 \mathrm{~L} \mathrm{Ar}$ per min for plasma gas, 1.45 L Ar per min for auxiliary gas and $1.14 \mathrm{~L}$ Ar per min for the sample gas at a RF power of $1260 \mathrm{~W}$. The measurements were carried out in medium resolution with determination of the following isotopes: ${ }^{55} \mathrm{Mn} ;{ }^{103} \mathrm{Rh}$. Before measurement, a so-called peak search was carried out to set the respective mass offset for each isotope for correct evaluation of masses. For calibration, a multielemental standard containing Mn was diluted with aqua bidest and a five point calibration with 0, 100, 250, 500 and $1000 \mu \mathrm{g} \mathrm{L}^{-1}$ containing $1 \mu \mathrm{g} \mathrm{L}^{-1} \mathrm{Rh}$ as the internal standard was carried out. The best calibrated isotopes (at least $R^{2}=0.999 \times$ ) were used for calculation of respective concentrations.

For determination of Fe levels in brain extracts and pellets ICP-OES analysis using an Optima 7300DV from Perkin Elmer was carried out. Therefore, extracts were diluted $1: 10$ with aqua bidest while acid digested pellets were measured undiluted. In both cases $100 \mu \mathrm{g} \mathrm{\textrm {L } ^ { - 1 }} \mathrm{Rh}$ served as the internal standard. Quantification was achieved by measurement of $100 \mu \mathrm{g} \mathrm{L} \mathrm{L}^{-1}$ certified multi-elemental standard. Operating parameters were the same as described above (see ICP-OES measurement of nitric acid digested whole brain).

Accuracy of ICP-sf-MS and ICP-OES measurements and comparability between both instruments were checked by analyzing certified reference material (bovine liver, BCR 185; $n=3$; certified values: Mn $9.3 \pm 0.3 \mu \mathrm{g} \mathrm{g}^{-1}$ ). Mn was determined at $8.1 \pm 0.08 \mu \mathrm{g} \mathrm{g}^{-1}$ or $8.49 \pm 0.23 \mu \mathrm{g} \mathrm{g}^{-1}$ by ICP-sf-MS or ICP-OES, respectively. Measured element levels on both instruments thus were comparable.

\section{Measurement of AchE activity in brain extracts}

The AchE Fluorescent Activity Kit of Arbor Assays (Michigan, US) was used for determination of AchE activity in the aqueous brain extracts. Therefore, samples were diluted 1:10 with the assay buffer and sonicated on ice for 30 seconds. $100 \mu \mathrm{L}$ of each sample and standard were transferred into a 96-well plate in duplets and $50 \mu \mathrm{L}$ of the reaction mix was added according to the distributor's manual. After incubation for 20 minutes at room temperature, the fluorescent intensity was determined at $510 \mathrm{~nm}$ after excitation at 370-410 $\mathrm{nm}$ with a conventional plate reader (Safire2, Tecan).

\section{Measurement of glutamate concentrations in brain extracts}

The Glutamate Assay Kit of BioVision (California, US) was used to determine glutamate concentrations in the aqueous brain extracts. Again, a 1:10 dilution of brain extracts in the supplied assay buffer was used for measurement. $50 \mu \mathrm{L}$ of samples or standards were added into a 96-well plate and $100 \mu \mathrm{L}$ of the 
reaction mix was added according to the distributor's manual. After incubating the plate at $37{ }^{\circ} \mathrm{C}$ for 30 minutes with the exclusion of light, the OD was measured at $450 \mathrm{~nm}$ using the plate reader mentioned above.

\section{IC-ICP-OES for determination of $\mathrm{Fe}(\mathrm{II}) /($ III) ratios}

Ion Chromatography hyphenated to ICP-OES was applied for separation of $\mathrm{Fe}(\mathrm{II})$ and $\mathrm{Fe}(\mathrm{III})$ in brain extracts. The used column was the PEEK guard column CG5A $(50 \times 4 \mathrm{~mm}$, Thermo, Germany) comprising a bilayer of anion- and cationexchange latex. The eluent consisted of $10 \mathrm{mM}$ Tris-HAc, $500 \mathrm{mM}$ $\mathrm{NH}_{4} \mathrm{Ac}$ and $1.5 \mathrm{mM}$ PDCA as the chelating agent with $\mathrm{pH}$ of 6.3 at an isocratic flow of $0.8 \mathrm{~mL} \mathrm{~min}^{-1}$ with $40 \% \mathrm{H}_{2} \mathrm{O}$. Eluent delivery was performed using the System Gold, 127 NM Solvent Module system from Beckmann, Germany, equipped with a Degassex ${ }^{\mathrm{TM}}$ mode DG 4490 (Phenomenex, Darmstadt, Germany) for degassing of eluents. The HPLC system was hyphenated to the ICP-OES by connecting the HPLC tube directly to the sample introduction tube of the nebulizer, therefore facilitating continuous introduction of the effluent of the column. Plasma settings for the ICP-OES system were the same as mentioned above except that RF power was set to $1350 \mathrm{~W}$ and the monitored spectral line for Fe detection was $237.562 \mathrm{~nm}$ as optimal parameters for detection settings.

$25 \mu \mathrm{L}$ of the extracts were injected via a syringe filter $(0.45 \mu \mathrm{m}$, MEMBREX, membraPure Membrantechnik, Reinstwasser, Germany) to filter off any residues, which might have influenced column operating conditions. The column was cleaned regularly with $1.5 \mathrm{mM} \mathrm{HCl}$ with subsequent re-equilibration of the column. Before and after each run, a blank run with $\mathrm{H}_{2} \mathrm{O}$ was carried out to ensure no carryover. Elution time and peak areas were calculated by the use of the Clarity Software, while peak areas were utilized for determination of the percentage of $\mathrm{Fe}($ II) and Fe(III) in the samples. Comparison with Fe standards yielded the retention times and was used for calculation of recovery. Therefore, $\mathrm{Fe}(\mathrm{II}) \mathrm{Cl}_{2} \cdot 4 \mathrm{H}_{2} \mathrm{O}$ standard solutions were prepared in aqua bidest at different concentrations $\left(100,200,300,400\right.$ and $\left.500 \mu \mathrm{g} \mathrm{L}^{-1}\right)$ both for external calibration as well as for matrix addition analysis for comparison. Fe(III)citrate served as the standard for $\mathrm{Fe}(\mathrm{III})$ determination by adding $\mathrm{HNO}_{3}$ to the standard solutions to ensure release of total $\mathrm{Fe}(\mathrm{III})$ from the citrate complex. Due to the need of adding $\mathrm{HNO}_{3}$ to the $\mathrm{Fe}$ (III) standard, no matrix addition could be carried out and external calibration by standard addition was used for calculation of recovery of Fe(III). Stock solutions of $1 \mathrm{~g} \mathrm{~L}^{-1}$ in aqua bidest and extraction buffer were prepared freshly before analysis. Regarding Fe(III), standard solutions of 100, 250, 400, 500 and $750 \mu \mathrm{g} \mathrm{L}^{-1}$ were prepared with $30 \% \mathrm{HNO}_{3}(\mathrm{~V} / \mathrm{V}$ compared to added volume of standard).

Quality control. Retention times of $\mathrm{Fe}(\mathrm{II})$ and $\mathrm{Fe}(\mathrm{III})$ were verified by the respective standard solutions $\left(\mathrm{FeCl}_{2} \cdot 4 \mathrm{H}_{2} \mathrm{O}\right.$ and $\mathrm{Fe}(\mathrm{III})$ citrate). LoD was calculated according to the $3 \sigma$ criteria, which was $6.33 \mu \mathrm{g} \mathrm{L^{-1 }}$ for $\mathrm{Fe}(\mathrm{III})$ and $9.11 \mu \mathrm{g} \mathrm{\textrm {L } ^ { - 1 }}$ for $\mathrm{Fe}(\mathrm{II})$. The recovery for $\mathrm{Fe}(\mathrm{III})$ was $43-66 \%$ while it was $83-105 \%$ for $\mathrm{Fe}(\mathrm{II})$.

\section{ESI-FT-ICR-MS measurement of brain extracts}

Ultra-high resolution mass spectra were acquired using a Fourier transform ion cyclotron resonance mass spectrometer (ICR-FTMS,
Solarix, Bruker, Bremen, Germany) equipped with a 12 T superconducting magnet (Magnex Scientific, Varian Inc., Oxford, UK) and an electrospray source (ESI, Apollo II; Bruker Daltonics, Bremen, Germany). Measurements and external calibration were performed in the negative ionization mode with a liquid flow rate of $2 \mu \mathrm{L} \min ^{-1}$ and a temperature of $180{ }^{\circ} \mathrm{C}$. The ESI voltage difference between the electrode and the counter electrode was $3500 \mathrm{~V}$. An additional voltage drop of $500 \mathrm{~V}$ was maintained between the counter electrode and an inner cone inside the electrospray to further accelerate the ions toward the mass spectrometer. The dry gas flow rate was maintained at $4 \mathrm{~L} \mathrm{~min}^{-1}$ and the ESI nebulizer gas flow rate was kept at $2 \mathrm{~L} \mathrm{~min}^{-1}$. The spectra were recorded in a mass-to-charge-ratio $(\mathrm{m} / \mathrm{z})$ range of $123-1000$ with an ion accumulation time of $300 \mathrm{~ms}$ for better sensitivity.

ICR-FT-MS spectra were calibrated internally by using the exact masses of known rat brain metabolites. Calibration was performed using the data analysis software 4.1 (Bruker Daltonics, Bremen, Germany) and exported to peak lists at a signal to noise ratio $(\mathrm{S} / \mathrm{N})$ of 4 . After calibration, mass lists were uploaded to MassTRIX web server in order to perform a subsequent annotation of masses within an error range of one ppm.

\section{Statistical analysis and chromatogram data handling}

Statistical analysis was done by applying Student's $t$-test with $p$-values $<0.05$ considered to be statistically significant (*/\#p<0.05, $\left.{ }^{* *} p<0.01,{ }^{* *} p<0.001\right)$. Peak areas and retention times of analytes were processed using Clarity software and for better visualization using Peak Fit ${ }^{\mathrm{TM}}$ software version 4.11.

\section{Results and discussion}

\section{Mn and Fe levels in brain extracts, pellets and total brain}

Three weeks old rats were fed with either $500 \mathrm{mg} \mathrm{kg}^{-1} \mathrm{Mn}$ in the test group or $23 \mathrm{mg} \mathrm{kg}^{-1} \mathrm{Mn}$ serving as the control group (Co) for 53 days. For determination of elemental concentrations in the whole brain, nitric acid digestion was carried out, followed by measurement with ICP-OES. Furthermore, elemental concentrations in brain extracts and pellets were determined by ICP-sf-MS. Results are shown in Fig. 1A. In total, Mn was significantly increased $(p<0.05)$ in the test group by $17 \%$ (429 $\mathrm{ng} \mathrm{g}^{-1}$ brain in test rats compared to $368 \mathrm{ng} \mathrm{g}^{-1}$ brain in control rats). The extraction of $\mathrm{Mn}$ showed a significant increase $(p<0.001)$ in Mn concentrations in test rat brain extracts of $19 \%$ (137 $\mathrm{ng} \mathrm{g}^{-1}$ brain in test rats compared to $115 \mathrm{ng} \mathrm{g}^{-1}$ brain in control rats). In the pellets obtained during the extraction procedure, Mn was again significantly increased $(p<0.01)$ in test rat pellets by $16 \%$ (293 $\mathrm{ng} \mathrm{g}^{-1}$ brain in test rats compared to $253 \mathrm{ng} \mathrm{g}^{-1}$ brain in control rats). The extraction efficiency was determined by the quotient of the elemental concentration in extracts versus the total Mn concentration in the brain tissue obtained by nitric acid digestion. Hence, the extraction efficiency was $31.5 \pm 3.4 \%$ for control samples and $32.7 \pm 4.9 \%$ for test samples. The recovery was determined by the quotient of the sum of $\mathrm{Mn}$ concentration in extracts and pellets by the Mn concentration in the total brain tissue and was averaged 

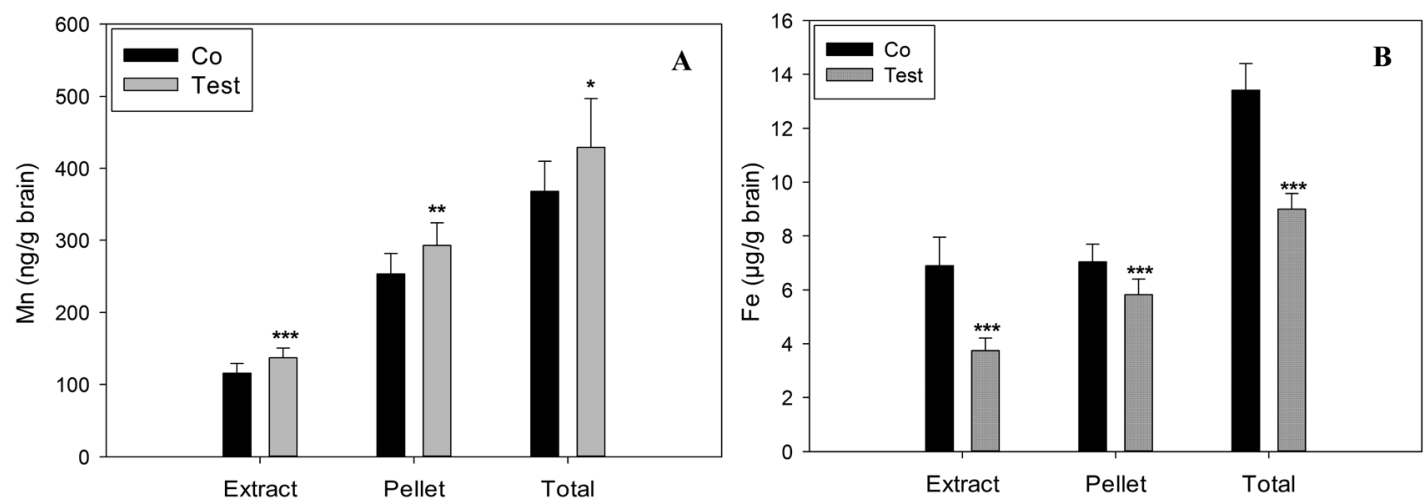

Fig. $1 \mathrm{Mn}$ and Fe concentrations in brain extracts, pellets and whole brain. Mn was significantly elevated (A) and Fe was significantly decreased (B) in test rat brain extracts ( $n=12$ extracts per group, 2 extracts per rat brain) and pellets $(n=12)$ as well as in whole brain (total, $n=18$ digestions per group, 3 digestions per rat brain) compared to control samples (Co). (mean $\pm \mathrm{SD}$, *** $p<0.001$, ${ }^{\star \star} p<0.01,{ }^{*} p<0.05$ ).

$99.9 \pm 6.4 \%$ (control: $98.1 \pm 6.7 \%$, test: $101.8 \pm 6.2 \%$ ). Thus, our results suggest that the applied feeding model was sufficient to elevate Mn concentrations in the test rat brains and further that the selective extraction procedures of Mn were highly efficient so that the extracts could be used for future analyses. For comparison to the results of the cited method used for extraction herein: there extraction efficiency for Mn was $26.6 \pm$ $1.5 \%{ }^{26}$

The results for measurement of Fe levels in the brain extracts and pellets as well as in the total brain, obtained in parallel by ICP-OES and ICP-sf-MS, are depicted in Fig. 1B. Total Fe concentrations were $8.9 \mu \mathrm{g} \mathrm{g}^{-1}$ brain for test rats and $13.4 \mu \mathrm{g} \mathrm{g}^{-1}$ brain for control rats. In a similar way, the concentrations in brain extracts and pellets were significantly decreased in test rats compared to those of control rats as well (extract: $3.7 \mathrm{ng} \mathrm{g}^{-1}$ brain compared to $6.9 \mathrm{ng} \mathrm{g}^{-1}$ brain in control rats; pellets: $5.8 \mathrm{ng} \mathrm{\textrm {g } ^ { - 1 }}$ brain compared to $7.0 \mathrm{ng} \mathrm{g}^{-1}$ brain in control rats). Calculation of extraction efficiency showed $48.9 \pm 5.9 \%$ for control samples and $41.5 \pm 3.8 \%$ for test samples. The recovery, calculated as described above, was averaged at $101.8 \pm 5.8 \%$ (control: $99.2 \pm 6.5 \%$, test: $104.4 \pm$ $5.1 \%)$. Hence, the extraction procedure was also specific for Fe.

\section{Evaluation of oxidative stress in the brain tissue}

AchE activity. Recent studies reported that Mn accumulation has an impact on AchE activity ${ }^{27,28}$ and thus we determined the latter in the rat brain extracts.

AchE activity was significantly increased by $9 \%$ in test rat brain extracts ( $979 \mathrm{mU} \mathrm{mL}^{-1} \mathrm{~g}^{-1}$ brain in test rats compared to $896 \mathrm{mU} \mathrm{mL}^{-1} \mathrm{~g}^{-1}$ brain in control rats) as shown in Fig. 2A.

The increase in brain AchE activity is consistent with other studies of sub-acute treatment with $\mathrm{Mn}^{27,29}$ For example, Chtourou et al. showed in a recent study that treatment of adult male Wistar rats with $20 \mathrm{mg} \mathrm{mL}^{-1} \mathrm{Mn}$ in drinking water for 30 days resulted in a significant increase in AchE activity as well as a considerable increase in AchE expression in cerebellum. ${ }^{28}$ In contrast, long-term or chronic treatment of rats with $\mathrm{Mn}$ in drinking water (e.g. $1 \mathrm{mg} \mathrm{mL}^{-1}$ for 2 years ${ }^{30}$ or $10 \mathrm{mg} \mathrm{mL}{ }^{-1}$ for 8 months $^{31}$ ) resulted in significant decreases of AchE activity. Summarizing these findings from the literature it appears that
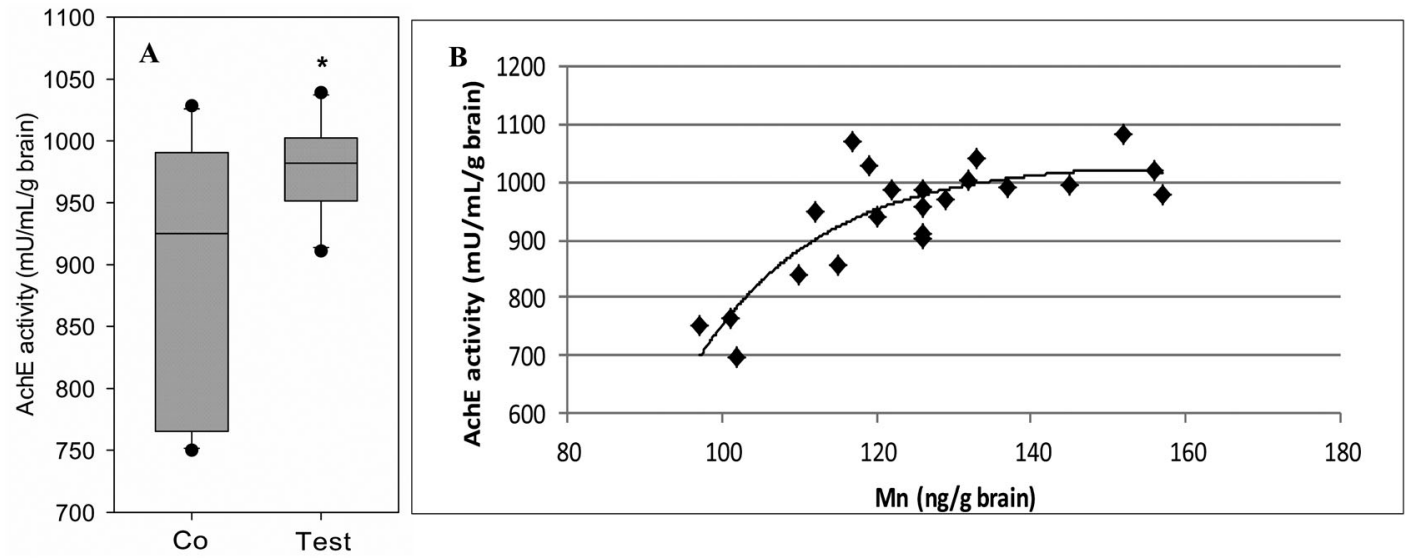

Fig. 2 AchE activity in brain extracts. (A) AchE activity was significantly increased in test rat brain extracts compared to control samples (Co) $(n=12$ extracts per group, * $p<0.05)$. (B) AchE activity was positively correlated with $\mathrm{Mn}$ concentrations in brain extracts $(n=22$ extracts, 11 per group) $\left(R^{2}=0.7194\right)$ showing an exponential rise to a certain maximum. 
AchE increases first as an acute response to $\mathrm{Mn}$, however, after adoption of the organism a decrease after prolonged exposure periods is observed. As the feeding duration in our study was 53 days, the observed enhancement of AchE activity might still be a response to the acute $\mathrm{Mn}$-induced oxidative stress in the test rat brains. However, the time frame in this study might present the edge between acute and chronic exposure compared to the time frame of the cited studies, explaining the only slight elevation of $9 \%$. Possibly, a high response to the oral Mn substitution was already flattened at the time point of measurement.

However, we obtained a positive, exponential correlation $\left(R^{2}=0.7194\right)$ between AchE activity and increasing Mn concentrations, measured in brain extracts. The correlation indicates an upper limit of AchE activity (around $1000 \mathrm{mU} \mathrm{mL}^{-1} \mathrm{~g}^{-1}$ brain) occurring from Mn concentrations of $130 \mathrm{ng} \mathrm{g}^{-1}$ brain (Fig. 2B). This leads to the suggestion that above a certain Mn concentration, AchE activity is attenuated, maybe due to the inability to compensate for Mn-induced intoxication.

Taken together, our results indicate a Mn-dependent significant increase in AchE activity in the rat brain as a sign of antioxidative response to Mn-induced oxidative stress. Such a stress-induced increase in AchE activity was also reported in $\mathrm{AD}$ as well as by Melo et al., who observed an enhanced AchE activity in cultured cells due to oxidative stress. ${ }^{32,33}$

Glutamate concentrations. Besides AchE activity, glutamate levels also seem to be altered by $\mathrm{Mn}$ accumulation in the brain. Therefore we also determined glutamate concentrations in the rat brain extracts.

In Fig. 3A the results for measurement of glutamate concentrations in the brain extracts are depicted. In the test rat brain extracts, glutamate was significantly increased by $19 \%$ (2.21 $\mathrm{mM} \mathrm{g}^{-1}$ brain compared to $1.85 \mathrm{mM} \mathrm{g}^{-1}$ brain).

Furthermore, correlation of glutamate with Mn concentrations in brain extracts indicated a linear, positive correlation $\left(R^{2}=0.4164\right)$ suggesting a Mn-dependent increase in glutamate concentrations (see Fig. 3B).
$\mathrm{Mn}$ is known to accumulate primarily in astrocytes, which are the most abundant cells in the CNS (appr. 50\% of volume) and facilitate numerous essential functions like glutamate homeostasis, where $\mathrm{Mn}$ is a cofactor of the glutamine synthetase. ${ }^{34}$ Here, we observed and confirmed an accumulation of glutamate, presumably derived through $\mathrm{Mn}$ inhibition of glutamine synthetase, that was reported before. ${ }^{35}$ During excessive Mn accumulation in cultured rat astrocytes, glutamate transporters (GLAST and taurine receptor) were found to be impaired. ${ }^{36}$ A down regulation of expression and function of glutamate transporters led to a reduced glutamate uptake and an increased extracellular glutamate level in rat neonatal primary astrocytes, which then provoked excitatory neurotoxicity. ${ }^{37}$ Both, extracellular $\left(198 \%, 150 \mu \mathrm{M} \mathbf{M n C l}_{2}\right)$ and intracellular $\left(170 \%, 300 \mu \mathrm{M} \mathrm{MnCl}_{2}\right)$ glutamate levels were increased by a low-level Mn exposure to AF5 rat neural-derived cells, ${ }^{38}$ which is consistent with the outcome of other studies, where an Mn-induced increase in intracellular and extracellular glutamate levels was observed in astrocytes. ${ }^{39,40}$ Despite these detailed Mn-induced mechanisms on the molecular level of glutamate homeostasis, knowledge about glutamate levels in rat brain tissue is still conflicting. On the one hand, Lipe et al. applied $20 \mathrm{mg} \mathrm{Mn}$ per $\mathrm{kg}$ per $\mathrm{d}$ in rats leading to a significant increase of $\mathrm{Mn}$ and glutamate concentrations in the rat brain, ${ }^{41}$ while on the other hand exposure to $6 \mathrm{mg} \mathrm{Mn}$ per $\mathrm{kg}$ per $\mathrm{d}$ led to a decrease in glutamate concentrations. ${ }^{42}$ Therefore, it seems that lower $\mathrm{Mn}$ exposure results in a decrease of glutamate, while high $\mathrm{Mn}$ concentrations result in an increase of glutamate concentrations in the brain. ${ }^{14}$ In summary, our findings reinforce the results of the cellular models with an increase in glutamate concentrations due to Mn-substitution. In this context, glutamate is presumably contributing to the enhancement of oxidative stress by activation of glutamate-gated cation channels in neurodegenerative occasions in general, e.g. PD or Alzheimer's Disease. $^{22}$ Elevated glutamate concentrations might also be a consequence of a NO-mediated mechanism, as NO was shown to
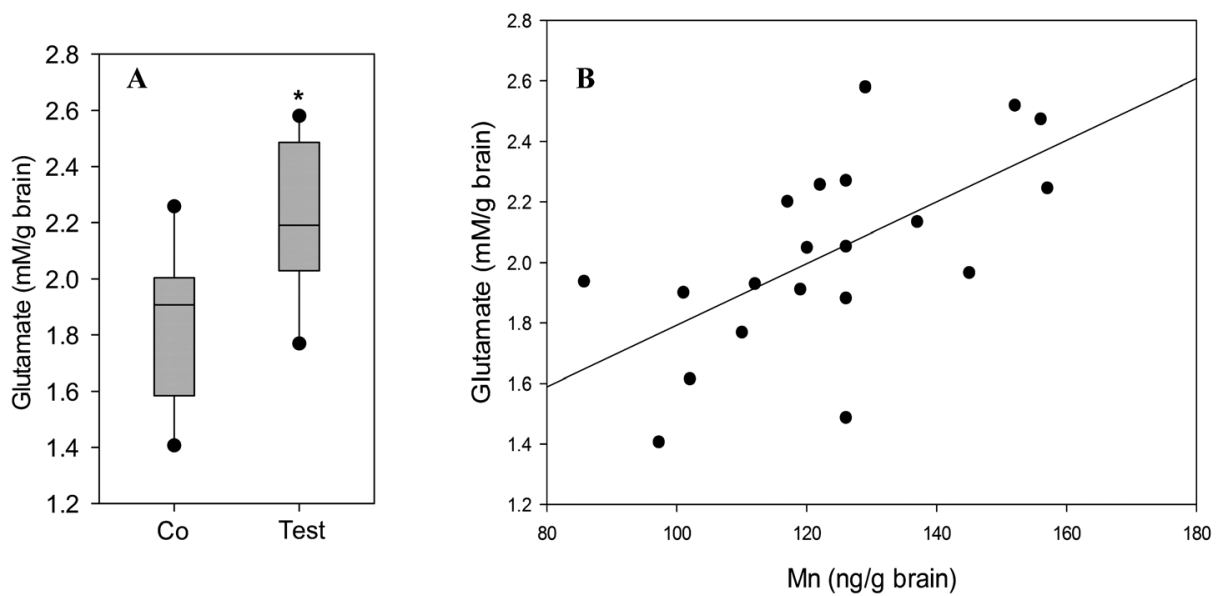

Fig. 3 Glutamate concentrations in brain extracts. (A) Glutamate concentrations were significantly increased in test rat brain extracts compared to control samples (Co) $(n=12$ extracts per group, * $p<0.05)$. (B) Glutamate concentrations were positively correlated with Mn concentrations in brain extracts $\left(R^{2}=0.4164\right)(n=20$ extracts, 10 per group). 
increase the release of glutamate from glia and neurons leading to excitotoxicity. ${ }^{43}$

ESI-FT-ICR-MS measurement. A targeted FT-ICR-MS analysis served as additional investigation of possible neurotoxic events by searching for markers of oxidative stress or signs of neuroinflammation due to Mn overexposure. A first evaluation based on differences in intensities of $\mathrm{m} / \mathrm{z}$ values showed four remarkable results: glutathione disulfide (GSSG) was significantly increased in test rat brain extracts 1.7-fold. Moreover, we observed two different prostaglandins, with significantly higher levels of PGB1 (detected only in test rats), PGH2 (5.8-fold increase) and additionally 15(S)HETE (15-hydroxyeicosatetraenoic acid) (27.9-fold increase) in brain extracts of test rats (Fig. $4 \mathrm{~A}-\mathrm{D})$.

15(S)-HETE together with 12(S)-HETE are the metabolites of 12/15-lipoxygenase (12/15-LOX), one of several lipid-peroxidizing enzymes present in the plant and animal kingdoms. ${ }^{44}$ In general, LOXs insert molecular oxygen into free and esterified polyunsaturated fatty acids. ${ }^{45}$ So far, 12/15-LOX has been described mainly in neurons and also in some glial cells of the cerebrum, basal ganglia, and hippocampus. ${ }^{46}$ Interestingly, an increase in its metabolic products was not only observed in brain ischemiareperfusion injury, ${ }^{47}$ but also in the brain of patients suffering from $\mathrm{AD},{ }^{48}$ suggesting an involvement of these metabolites in neurodegenerative disorders. Thus, 12/15-LOX might oxidize fatty acids in the cell membranes and therefore contribute to in vivo oxidative stress. ${ }^{49}$ The observed increase in 15(S)-HETE could be due to the Mn-induced formation of ROS in the brain, through direct activation of 12/15-LOX or by reaction of ROS products like $\mathrm{H}_{2} \mathrm{O}_{2}$ with $\mathrm{Fe}(\mathrm{II})$, which also contributes to lipid peroxidation. ${ }^{50}$ Furthermore, an activation of LOX was not only observed due to oxidative stress but also by interaction with NO. ${ }^{51}$ Therefore, the herein applied Mn substitution might contribute to a release of NO by activation of the inducible nitric oxide synthase in astrocytes ${ }^{52}$ leading to the assumption of a NO-mediated mechanism of Mn-induced oxidative stress in the brain. ${ }^{53}$

Another sign for inflammatory processes in the brain is the detected increase of the two prostaglandins PGH2 and PGB1, while the latter was only detected in the Mn-treated group. One of many responses to inflammation is the release of arachidonic acid and its conversion to proinflammatory prostaglandins. ${ }^{54}$ PGB1 is the non-enzymatic dehydration product of PGE1 and a metabolite of PBA1, which is known to modify the pressor responses to sympathetic nerve stimulation. ${ }^{55} \mathrm{PGH} 2$ is the intermediate product in the synthesis of prostaglandins, which is produced from arachidonic acid and is the precursor metabolite for the synthesis of the prostaglandins from the I, E, F and D group. A disturbed arachidonic acid metabolism is - besides the mitochondrial respiratory chain and NADPH oxidase - the major source of ROS. ${ }^{56}$ Hence, the herein observed upregulated prostaglandins represent major proinflammatory lipid mediators ${ }^{57}$ and are a further sign of oxidative stress in the brain due to $\mathrm{Mn}$ substitution. Similar results were obtained by Santos et al., who observed an increase in PGE2 in rats, which received $\mathrm{MnCl}_{2}$ injections. $^{58}$

Oxidative stress is further characterized by a depletion of $\mathrm{GSH}^{57}$ and an increase in GSSG. ${ }^{59}$ In our study, a specific decrease in GSH could not be detected using the applied method, but interestingly GSSG was significantly increased in Mn-treated rats. Therefore, we assume that due to the oxidative stress, the oxidized form of GSH (namely GSSG) is prevalently formed in the neuronal site. This in turn is a further hint for Mn-induced oxidative stress in the brain. Hernandez et al. also were able to observe an increase in GSH at low Mn concentrations but a decrease in GSH at higher levels of $\mathrm{Mn}$ in two different cell lines in vitro, ${ }^{60}$ presumably by shifting the balance towards GSSG.

These results of ESI-FT-ICR-MS all contribute to the supposed induction of oxidative stress due to Mn. Furthermore, they reveal deeper insight into the different mechanisms, which are discussed in the process of neurodegeneration due to $\mathrm{Mn}$ (see also Fig. 7).

\section{Determination of $\mathrm{Fe}(\mathrm{II}) /(\mathrm{III})$-ratios in brain extracts}

To investigate possible differences in concentrations of $\mathrm{Fe}(\mathrm{II})$ or $\mathrm{Fe}(\mathrm{III})$ in the brain of control and test rats, ion chromatography

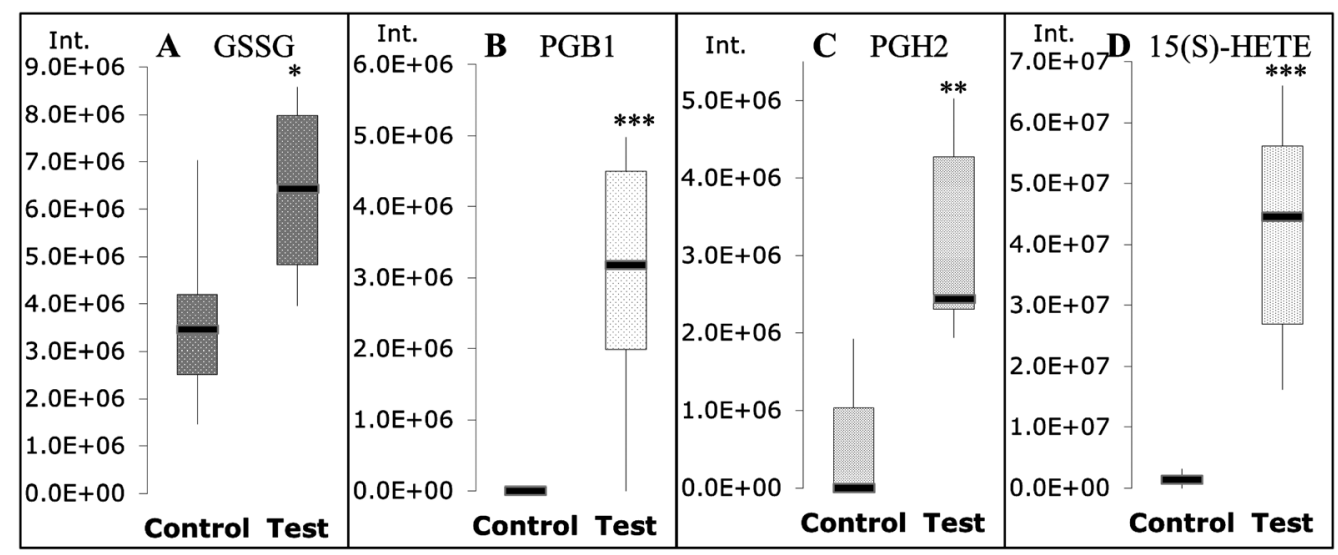

Fig. 4 Most prominent upregulated markers of oxidative stress. Based on differences in intensities of FT-ICR-MS measurement GSSG was 1.7-fold $(A, p=0.04)$, PGB1 was only detected in test samples $(B, p=0.0001), P G H 2$ was 5.8 -fold $(C, p=0.003)$ and $15(S)$-HETE was 27.9-fold (D, $p=0.0006)$ increased in test rat brain extracts compared to control samples (MeOH extracts, $n=6$ extracts per group). 

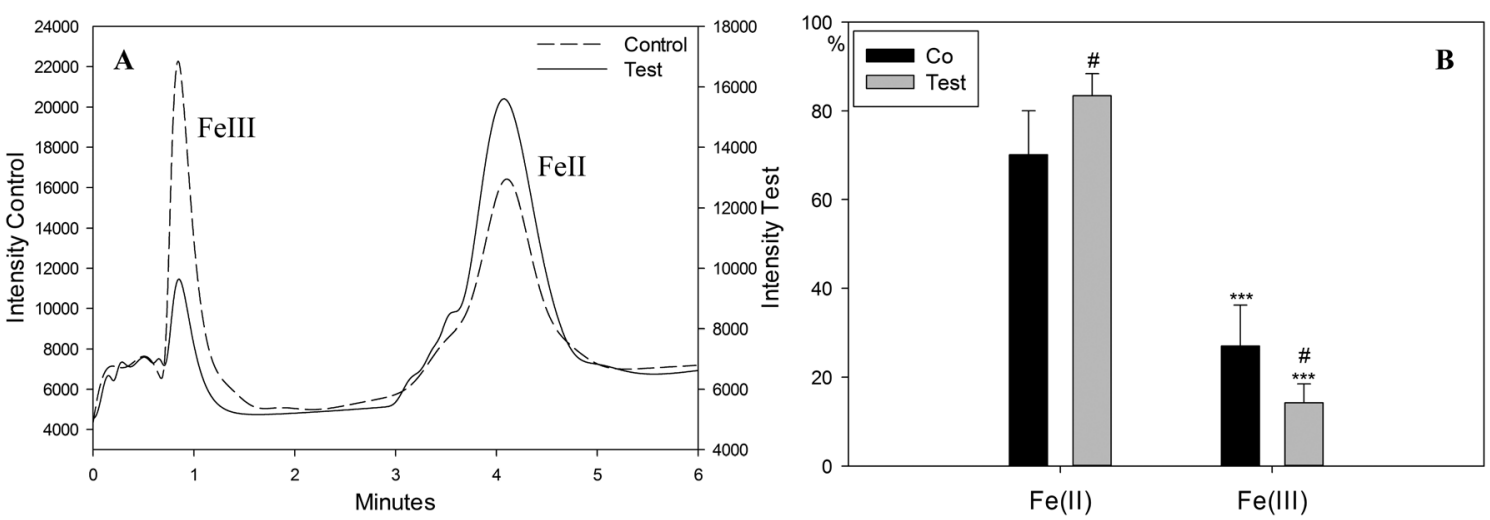

Fig. 5 Determination of $\mathrm{Fe}(\mathrm{II}) /(\mathrm{II})$ ratios in brain extracts by IC-ICP-OES. (A) Examples for Fe(II)/(III)-chromatograms of control and test rat brain extracts show elution of $\mathrm{Fe}(\mathrm{III})$ and $\mathrm{Fe}(I)$ at 0.8 and 4.1 minutes respectively; data were processed using PeakFit software Version 4.11. (B) Differences (area percent)

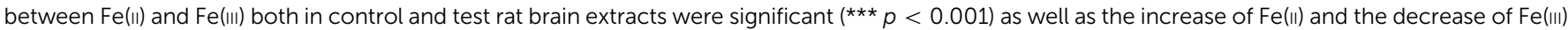
area in test rat brain extracts compared to control rat brain extracts $(C o, \# p<0.05)$ were obtained as significant $(n=12$ extracts per group, mean \pm SD).

was hyphenated to ICP-OES (IC-ICP-OES) for separation and detection of Fe in the brain extracts. Representative examples of chromatograms for control and test samples are shown in Fig. 5A. A remarkable difference in concentrations of $\mathrm{Fe}(\mathrm{II})$ and $\mathrm{Fe}(\mathrm{III})$ in the control and the test sample was observed: The Fe(II) peak area (at 4.1 minutes) of the test sample was increased while the $\mathrm{Fe}$ (III) peak area (at 0.8 minutes) was decreased, when compared to the control sample.

After analyzing all brain extracts, we observed a significant ( $p=0.015)$ shift of $\mathrm{Fe}(\mathrm{II}) /(\mathrm{III})$-homeostasis towards $\mathrm{Fe}(\mathrm{II})$ in the test rat brain extracts by comparison of area percent as shown in Fig. 5B.

The obtained concentrations of $\mathrm{Fe}(\mathrm{II})$ and $\mathrm{Fe}(\mathrm{III})$ were correlated with $\mathrm{Mn}$ concentrations in the brain extracts as shown in Fig. 6.

The correlation of $\mathrm{Fe}(\mathrm{II})$ showed a stronger, linear correlation with $\mathrm{Mn}$ concentrations in the brain extracts compared to the correlation of $\mathrm{Fe}$ (III) in both control (Fig. 6A) and test (Fig. 6B) rat brain extracts. This correlation was even slightly stronger in the test rat brain extracts $\left(R^{2}=0.6574\right.$ for test samples compared to $R^{2}=0.5714$ for control samples). The correlation between $\mathrm{Fe}(\mathrm{II})$ and $\mathrm{Mn}$ was statistically significant both in control and test samples ( $p=0.018$ and $p=0.008$, respectively). Correlation of Fe(III) with Mn showed no statistical significance both in control and test samples $(p=0.109$ and $p=0.074$, respectively). According to the slope of the trendline, these data indicate a stronger increase in $\mathrm{Fe}(\mathrm{II})$ (control: $y=41.465 x+$ 147.66, $R^{2}=0.5714$; test: $\left.y=25.226 x+67.492, R^{2}=0.6574\right)$ than Fe(III) (control: $y=19.691 x-26.062, R^{2}=0.3256$; test: $y=$ $10.254 x-64.345, R^{2}=0.3869$ ) with higher Mn concentration in the rat brain indicating a misbalancing of the $\mathrm{Fe}(\mathrm{II}) / \mathrm{Fe}(\mathrm{III})$ redox couple.

Since Fe is a further "biometal", which is a prerequisite of neurons, an abnormal distribution of Fe can lead to different brain injuries. Therefore, excessive Fe deposition in the CNS was observed in neurodegenerative diseases like Alzheimer's Disease, ALS and also PD. ${ }^{18,19,61}$ Acute brain injuries, like ischemia also lead to a release of Fe out of mitochondrial heme into neurons. ${ }^{62}$ In more detail, the degradation of heme will contribute to a release of $\mathrm{Fe}(\mathrm{II})$, which may then generate free radicals in the Fenton reaction and therefore lead to neuronal injury or damage. ${ }^{22,62}$ This possible mechanism of Fe-dependent neuronal damage is one of several proposed mechanisms. In the case of ischemia, the inhibition of detoxifying enzymes like SOD or glutathione peroxidase causes prevention of oxidation of $\mathrm{Fe}$ (II) to $\mathrm{Fe}(\mathrm{III}) .{ }^{62} \mathrm{~A}$ missing increase in the expression of ferritin leads to an inhibited removal of the increased $\mathrm{Fe}(\mathrm{II}) .{ }^{63}$ Both mechanisms thus promote $\mathrm{Fe}(\mathrm{II})$ accumulation. Especially the lack of removal of $\mathrm{Fe}(\mathrm{II})$ from neurons might also be the case in our study, which could be due to a competition for metal transporter binding as the body is engaged with neuronal clearing of Mn. Therefore, enzymes which are usually complexing Fe might now be occupied with Mn due to the need of removal of Mn. This in turn leads to an increase in the highly reactive free iron, which can then prompt oxidative stress via the Fenton reaction as shown in eqn (1):

$$
\mathrm{Fe}(\mathrm{II})+\mathrm{H}_{2} \mathrm{O}_{2} \rightleftharpoons \mathrm{FeOH}^{2+}+\cdot \mathrm{OH}
$$

Interestingly, $\mathrm{H}_{2} \mathrm{O}_{2}$ can not only act as an oxidizing but rather as a reducing agent, prompting the reduction of $\mathrm{Fe}(\mathrm{III})$ to $\mathrm{Fe}(\mathrm{II}):^{64}$

$$
\begin{gathered}
\mathrm{Fe}(\mathrm{III})+\mathrm{H}_{2} \mathrm{O}_{2} \rightleftharpoons \mathrm{Fe}(\mathrm{II})+\mathrm{O}_{2}{ }^{-}+2 \mathrm{H}^{+} \\
\mathrm{Fe}(\mathrm{III})+\mathrm{O}_{2}{ }^{-} \rightleftharpoons \mathrm{Fe}(\mathrm{II})+\mathrm{O}_{2}
\end{gathered}
$$

By assuming that ROS like $\mathrm{H}_{2} \mathrm{O}_{2}$ is already present in the brain tissue due to $\mathrm{Mn}$ intoxication ${ }^{58,65-67}$ this $\mathrm{H}_{2} \mathrm{O}_{2}$ might then react with the $\mathrm{Fe}(\mathrm{III})$ and the resulting $\mathrm{Fe}(\mathrm{II})$ might then further react via the Fenton reaction and produce more ROS (eqn (2)) or the resulting $\mathrm{O}_{2}{ }^{-}$then further reduces more $\mathrm{Fe}(\mathrm{III})$ (eqn (3)), producing even more $\mathrm{Fe}(\mathrm{II})$. This process might describe a vicious circle, where $\mathrm{Mn}$ might lead to the first step of generation of ROS and building a positive feedback loop by Fe-mediated ROS generation. ${ }^{64}$

Due to the similarities in molecular mechanisms of manganism to PD, a study in a PD-model in monkeys is of special interest. 

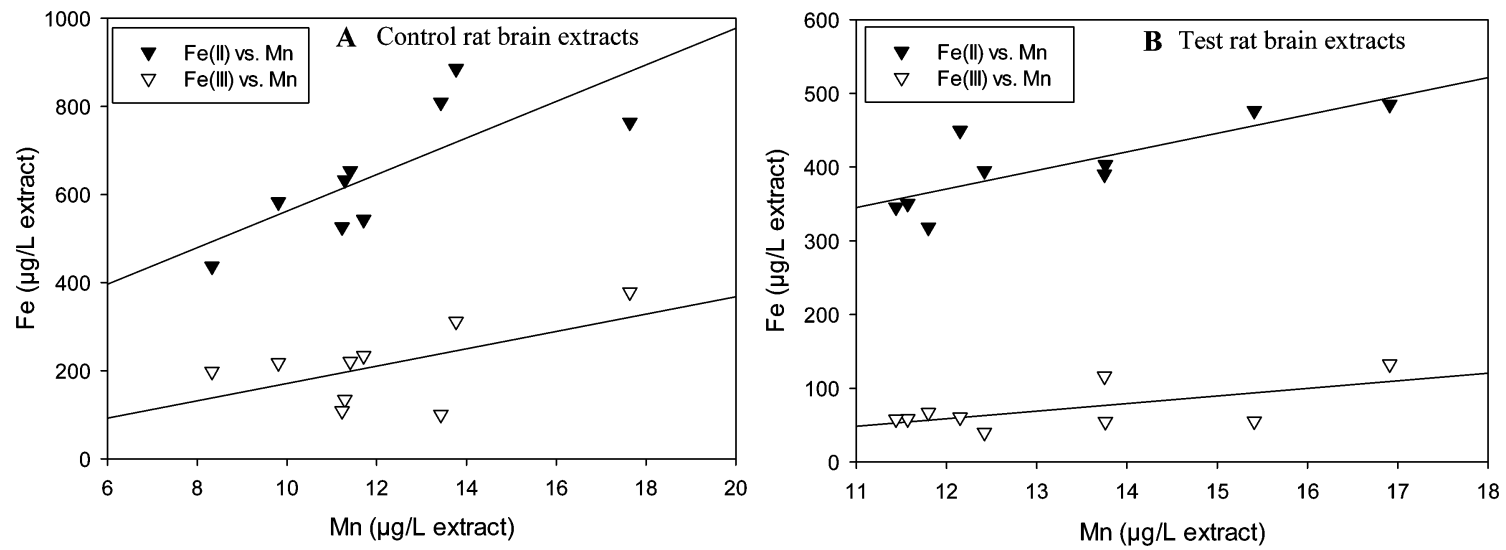

Fig. 6 Correlation of Fe(II) and Fe(III) with Mn concentrations in rat brain extracts. (A) Fe(II) was stronger positively correlated $\left(y=41.465 x+147.66, R^{2}=0.5714\right)$ with $\mathrm{Mn}$ concentrations in control rat brain extracts than Fe(III) $\left(y=19.691 x-26.062, R^{2}=0.3256\right)(n=9$ extracts). (B) Fe(II) was stronger positively correlated $\left(y=25.226 x+67.492, R^{2}=0.6574\right)$ with Mn concentrations in test rat brain extracts than Fe(III) $\left(y=10.254 x-64.345, R^{2}=0.3869\right)(n=9$ extracts).

Therein, the induction of PD lead to an infiltration of Fe-containing microglia in the substantia nigra pars compacta, which was associated with the disappearance of dopaminergic neurons. ${ }^{68}$ Interestingly, also in PD no ferritin is found in neurons of substantia nigra but vicinal to dopaminergic neurons an abundance of reactive, ferritin-positive microglia were observed. ${ }^{69}$ These studies indicate that accumulation of $\mathrm{Fe}$ in neurodegeneration might arise from accumulation of Fe-containing inflammatory cells. $^{62}$ These mechanisms of Fe-accumulation found in PD might also be true for manganism scenarios, so that Fe accumulates in or near dopaminergic neurons, is not transported and cannot be oxidized due to inactivity of antioxidative enzymes. The abundance of present $\mathrm{Fe}$ (II) can then react via the Fenton reaction by forming hydroxyl radicals leading to cell death. This can be indicated by our findings: initially, an increase in Mn concentrations results likely in

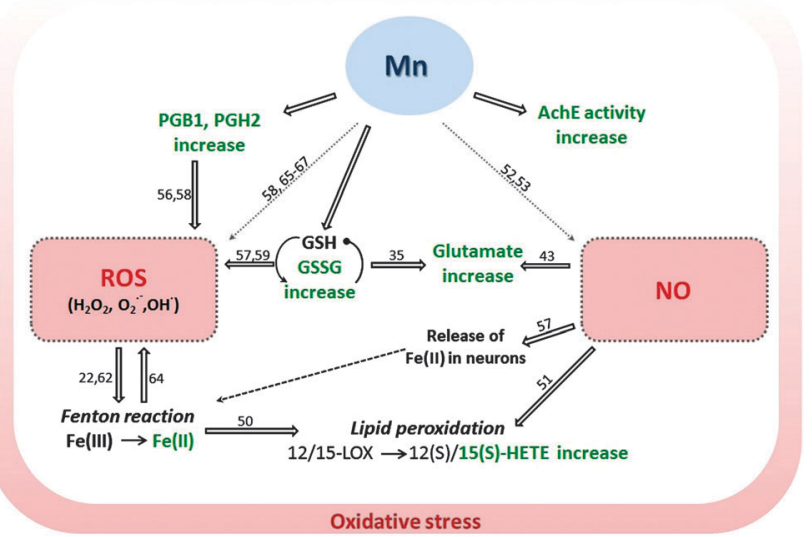

Fig. 7 Summary of results and supposed interaction of ongoing oxidative events during elevated $\mathrm{Mn}$ concentrations in the brain. The figure summarizes the supposed interaction of possible neurotoxic mechanisms due to $\mathrm{Mn}$ substitution combining the results of the investigations herein and of the literature (reference numbers on arrows). The upregulated markers or elements found in this research are shown in green. (ROS: reactive oxygen species, NO: nitric oxide, AchE: acetylcholinesterase, GSH: glutathione, GSSG: glutathione disulfide, PGB1/H2: prostaglandine B1/H2, 12/15-LOX: 12/15-lipoxygenase, 12(S)/15(S)-HETE: 15S-hydroxy-5Z,8Z,11Z,13E-eicosatetraenoic acid). an induction of oxidative stress, causing the shift in Fe-homeostasis in the brain and therefore enables the versatile Fe-induced neuronal injury mechanisms, which exaggerate the Mn induced neurodegenerative mechanism.

\section{Conclusion}

We herein investigated possible mechanisms for neuroinflammation resulting from an increased oral Mn supplementation in rats. The observed results are summarized in Fig. 7: increases in AchE activity, glutamate levels as well as GSSG in the brain of Mn treated rats are different signs of the ongoing oxidative stress. Furthermore, prostaglandins like PGB1 and PGH2 were elevated in the test samples, which are also known as proinflammatory lipid mediators. These might result from a disturbed arachidonic acid metabolism, which is in turn a major source of ROS triggering lipid peroxidation, underlined by the detection of elevated 15(S)-HETE in the brain of Mn treated rats in this study. IC-ICP-OES analysis of brain samples further revealed for the first time that Mn might induce a shift of the $\mathrm{Fe}(\mathrm{II}) /(\mathrm{III})$ ratio towards $\mathrm{Fe}(\mathrm{II})$, which could then participate in different oxidative reactions like the Fenton reaction or lipid peroxidation. Interestingly, Fe(II) was significantly correlated with $\mathrm{Mn}$ concentrations in the rat brain extracts, showing an increased level of $\mathrm{Fe}(\mathrm{II})$ accompanied with higher Mn concentrations. In summary, our results underline and deepen current knowledge about the interaction of the different mechanisms of oxidative stress in neuronal tissue due to an increased concentration of Mn.

\section{Conflicts of interest}

The authors declare no conflict of interest.

\section{Acknowledgements}

The authors thank Peter Grill for technical assistance and Franco Moritz for assistance with data evaluation. 


\section{References}

1 K. M. Erikson, T. Syversen, J. L. Aschner and M. Aschner, Environ. Toxicol. Pharmacol., 2005, 19, 415-421.

2 M. Aschner, T. R. Guilarte, J. S. Schneider and W. Zheng, Toxicol. Appl. Pharmacol., 2007, 221, 131-147.

3 M. Sidoryk-Wegrzynowicz and M. Aschner, J. Intern. Med., 2013, 273, 466-477.

4 B. A. Racette, L. McGee-Minnich, S. M. Moerlein, J. W. Mink, T. O. Videen and J. S. Perlmutter, Neurology, 2001, 56, 8-13.

5 B. A. Racette, S. D. Tabbal, D. Jennings, L. Good, J. S. Perlmutter and B. Evanoff, Neurology, 2005, 64, 230-235.

6 S. Zoni, G. Bonetti and R. Lucchini, J. Trace Elem. Med. Biol., 2012, 26, 179-182.

7 B. Michalke, A. Berthele, P. Mistriotis, M. OchsenkuhnPetropoulou and S. Halbach, Electrophoresis, 2007, 28, 1380-1386.

8 M. Bouchard, D. Mergler, M. Baldwin, M. Panisset and H. A. Roels, Neurotoxicology, 2007, 28, 290-297.

9 R. Lucchini and N. Zimmerman, Neurotoxicology, 2009, 30, 1144-1148.

10 J. Bornhorst, C. A. Wehe, S. Huwel, U. Karst, H.-J. Galla and T. Schwerdtle, J. Biol. Chem., 2012, 287, 17140-17151.

11 M. Aschner, K. M. Erikson, E. H. Hernandez and R. Tjalkens, NeuroMol. Med., 2009, 11, 252-266.

12 C. E. Gavin, K. K. Gunter and T. E. Gunter, Biochem. J., 1990, 266, 329-334.

13 C. E. Gavin, K. K. Gunter and T. E. Gunter, Neurotoxicology, 1999, 20, 445-453.

14 K. M. Erikson and M. Aschner, Neurochem. Int., 2003, 43, 475-480.

15 M. Carlsson and A. Carlsson, Trends Neurosci., 1990, 13, 272-276.

16 C. W. Cotman, A. Foster and T. Lanthorn, Adv. Biochem. Psychopharmacol., 1981, 27, 1-27.

17 E. S. Lee, M. Sidoryk, H. Jiang, Z. Yin and M. Aschner, J. Neurochem., 2009, 110, 530-544.

18 W. Y. Ong and A. A. Farooqui, J. Alzheimer's Dis., 2005, 8, 183-200; discussion 209-115.

19 C. Quintana, S. Bellefqih, J. Y. Laval, J. L. Guerquin-Kern, T. D. Wu, J. Avila, I. Ferrer, R. Arranz and C. Patino, J. Struct. Biol., 2006, 153, 42-54.

20 J. F. Schenck, J. Neurol. Sci., 2003, 207, 99-102.

21 S. M. Sadrzadeh and Y. Saffari, Am. J. Clin. Pathol., 2004, 121(suppl), S64-S70.

22 J. T. Coyle and P. Puttfarcken, Science, 1993, 262, 689-695.

23 K. A. Erikson, Z. K. Shihabi, J. L. Aschner and M. Aschner, Biol. Trace Elem. Res., 2002, 87, 143-156.

24 C. L. Kwik-Uribe, D. Gietzen, J. B. German, M. S. Golub and C. L. Keen, J. Nutr., 2000, 130, 2821-2830.

25 NRC, Nutrient Requirements of Laboratory Animals, Nat. Acad. Press., Washington, DC, 4th revised edn, 1995.

26 J. Diederich and B. Michalke, Anal. Bioanal. Chem., 2011, 399, 1799-1806.

27 C. Liapi, A. Zarros, P. Galanopoulou, S. Theocharis, N. Skandali, H. Al-Humadi, F. Anifantaki, E. Gkrouzman,
Z. Mellios and S. Tsakiris, Basic Clin. Pharmacol. Toxicol., 2008, 103, 171-175.

28 Y. Chtourou, H. Fetoui, E. M. Garoui, T. Boudawara and N. Zeghal, Neurochem. Res., 2012, 37, 469-479.

29 M. A. Lebda, M. S. El-Neweshy and Y. S. El-Sayed, Neurotoxicology, 2012, 33, 98-104.

30 J. C. Lai, T. K. Leung and L. Lim, J. Neurochem., 1981, 36, 1443-1448.

31 H. Martinez and E. Bonilla, Neurobehav. Toxicol. Teratol., 1981, 3, 277-280.

32 V. N. Talesa, Mech. Ageing Dev., 2001, 122, 1961-1969.

33 J. B. Melo, P. Agostinho and C. R. Oliveira, Neurosci. Res., 2003, 45, 117-127.

34 P. Karki, E. Lee and M. Aschner, Ann. Occup. Environ. Med., 2013, 25, 4.

35 Y. Deng, Z. Xu, B. Xu, D. Xu, Y. Tian and W. Feng, Biol. Trace Elem. Res., 2012, 148, 242-249.

36 K. Erikson and M. Aschner, Neurotoxicology, 2002, 23, 595-602.

37 M. Sidoryk-Wegrzynowicz, E. Lee, J. Albrecht and M. Aschner, J. Neurochem., 2009, 110, 822-830.

38 D. R. Crooks, N. Welch and D. R. Smith, Neurotoxicology, 2007, 28, 548-554.

39 C. Zwingmann, D. Leibfritz and A. S. Hazell, J. Neurochem., 2003, 87, 142.

40 L. Mutkus, J. L. Aschner, V. Fitsanakis and M. Aschner, Biol. Trace Elem. Res., 2005, 107, 221-230.

41 G. W. Lipe, H. Duhart, G. D. Newport, W. Slikker Jr. and S. F. Ali, J. Environ. Sci. Health, Part B, 1999, 34, 119-132.

42 J. C. Lai, T. K. Leung and L. Lim, Neurotoxicology, 1984, 5, 37-47.

43 G. C. Brown and A. Bal-Price, Mol. Neurobiol., 2003, 27, 325-355.

44 H. Kuhn and B. J. Thiele, FEBS Lett., 1999, 449, 7-11.

45 A. R. Brash, J. Biol. Chem., 1999, 274, 23679-23682.

46 M. Nishiyama, T. Watanabe, N. Ueda, H. Tsukamoto and K. Watanabe, J. Histochem. Cytochem., 1993, 41, 111-117.

47 L. Sautebin, C. Spagnuolo, C. Galli and G. Galli, Prostaglandins, 1978, 16, 985-988.

48 D. Pratico, V. Zhukareva, Y. Yao, K. Uryu, C. D. Funk, J. A. Lawson, J. Q. Trojanowski and V. M. Lee, Am. J. Pathol., 2004, 164, 1655-1662.

49 C. D. Funk and T. Cyrus, Trends Cardiovasc. Med., 2001, 11, 116-124.

50 A. A. Farooqui, Neurochemical Aspects of Neroinflammation in Brain in Molecular Aspects of Neurodegeneration and Neuroprotection, Bentham Science, Oak Park, Ill, USA, 2010.

51 K. Czubowicz, G. A. Czapski, M. Cieslik and R. P. Strosznajder, Folia Neuropathol., 2010, 48, 283-292.

52 M. Spranger, S. Schwab, S. Desiderato, E. Bonmann, D. Krieger and J. Fandrey, Exp. Neurol., 1998, 149, 277-283. 53 X. Liu, Toxicol. Sci., 2006, 91, 521-531.

54 A. A. Farooqui, L. A. Horrocks and T. Farooqui, J. Neurochem., 2007, 101, 577-599.

55 N. Himori and A. M. Burkman, Res. Commun. Chem. Pathol. Pharmacol., 1983, 41, 397-405. 
56 G. Y. Sun, L. A. Horrocks and A. A. Farooqui, J. Neurochem., 2007, 103, 1-16.

57 T. Farooqui and A. A. Farooqui, Parkinson's Dis., 2011, 2011, 247467.

58 D. Santos, D. Milatovic, V. Andrade, M. C. Batoreu, M. Aschner and A. P. Marreilha dos Santos, Toxicology, 2012, 292, 90-98.

59 S. Luperchio, S. Tamir and S. R. Tannenbaum, Free Radicals Biol. Med., 1996, 21, 513-519.

60 R. B. Hernandez, M. Farina, B. P. Esposito, N. C. SouzaPinto, F. Barbosa Jr. and C. Sunol, Toxicol. Sci., 2011, 124, 414-423.

61 G. A. Salvador, BioFactors, 2010, 36, 103-110.

62 T. Moos and E. H. Morgan, Ann. N. Y. Acad. Sci., 2004, 1012, 14-26.
63 M. D. Knutson, J. E. Levy, N. C. Andrews and M. WesslingResnick, J. Nutr., 2001, 131, 1459-1464.

64 D. C. Crans, K. A. Woll, K. Prusinskas, M. D. Johnson and E. Norkus, Inorg. Chem., 2013, 52, 12262-12275.

65 S. F. Ali, H. M. Duhart, G. D. Newport, G. W. Lipe and W. Slikker Jr., Neurodegeneration, 1995, 4, 329-334.

66 Y. Chtourou, K. Trabelsi, H. Fetoui, G. Mkannez, H. Kallel and N. Zeghal, Neurochem. Res., 2011, 36, 1546-1557.

67 A. W. Dobson, S. Weber, D. C. Dorman, L. K. Lash, K. M. Erikson and M. Aschner, Biol. Trace Elem. Res., 2003, 93, 113-126.

68 K. Goto, H. Mochizuki, H. Imai, H. Akiyama and Y. Mizuno, Brain Res., 1996, 724, 125-128.

69 B. Mirza, H. Hadberg, P. Thomsen and T. Moos, Neuroscience, 2000, 95, 425-432. 